\title{
Efecto de las insignias digitales en la actividad virtual de universitarios en modalidad semipresencial
}

\author{
Eduardo Fabio Gonzales López* \\ Universidad Tecnológica del Perú, Lima \\ ORCID https://orcid.org/0000-0002-4097-0613
}

Recibido: 16/09/2019; Revisado: 23/05/2019; Publicado: 15/10/2019

\begin{abstract}
Resumen
La presente investigación buscó determinar los efectos de un programa de insignias digitales sobre el desempeño académico en el curso Inglés I y la participación en las actividades virtuales. El diseño de investigación fue experimental con post-prueba y grupo control. La muestra estuvo conformada por 18 estudiantes del primer ciclo, matriculados en modalidad semipresencial en una universidad privada de Lima. Se encontraron diferencias estadísticamente significativas entre el grupo experimental y control, a favor del grupo experimental. Además, se halló un tamaño del efecto de 4.56, categorizado como muy grande. En el caso del rendimiento académico, no se encontraron diferencias estadísticamente significativas. Sin embargo, el tamaño del efecto fue 0.60, considerado como moderado. En conclusión, el programa de insignias digitales fomentó la participación en las actividades académicas virtuales; sin embargo, los resultados respecto del rendimiento académico no son totalmente claros, por lo que se sugiere realizar investigaciones en cursos de diferente área y con una muestra más amplia.
\end{abstract}

Palabras clave: Insignia digital, semipresencial, universidad, rendimiento académico

\section{Effects of Digital Badges on the Online Activity of University Students in a Blended Learning Model}

\begin{abstract}
This research aimed to determine the effects a digital badge program had on the academic performance and participation in online activities in the English I course. The research design was experimental with post-test and control group. The sample consisted of 18 students from the first semester, enrolled in a blended learning model in a private university in Lima. There were statistically significant differences between the control and experimental group, which favored the experimental group. In addition, an effect size of 4.56 was found, which was considered very large. Regarding academic performance, no statistically significant differences were found. However, the effect size was 0.60 , considered moderate. In conclusion, the digital badge program encouraged participation in virtual academic activities; however, the results obtained in connection with academic performance are not entirely clear, which is why it is suggested to conduct research in courses of different areas, with a broader sample.

Keywords: digital badge, blended learning, university, academic performance
\end{abstract}




\title{
Efeito dos distintivos digitais na atividade virtual de universitários na modalidade semipresencial
}

\begin{abstract}
Resumo
Esta pesquisa buscou determinar os efeitos de um programa de crachás digitais no desempenho acadêmico do curso de inglês I e na participação em atividades virtuais. O desenho da pesquisa foi experimental com pós-teste e grupo de controle. A amostra esteve composta por 18 estudantes do primeiro período, matriculados na modalidade semipresencial em uma universidade privada de Lima. Encontraram-se diferenças estatisticamente significativas entre o grupo experimental e de controle, a favor do grupo experimental. Outro achado foi um tamanho do efeito de 4,56, categorizado como muito grande. No caso do rendimento acadêmico, não se encontraram diferenças estatisticamente significativas. No entanto, o tamanho do efeito foi de 0.60 , considerado como moderado. Em conclusão, o programa de distintivos digitais fomentou a participação nas atividades virtuais; porém, os resultados obtidos a respeito do rendimento acadêmico não são totalmente claros, motivo pelo qual recomenda-se realizar pesquisas em cursos de área diferente e com uma amostra mais ampla.
\end{abstract}

Palavras chave: emblemas digitais, semipresencial, universidade, rendimento acadêmico

Como citar:

Gonzales, E. (2019). Efectos de las insignias digitales en la actividad virtual de universitarios en modalidad semipresencial. Revista Digital de Investigación en Docencia Universitaria, 13(2), 29 - 40. https://doi.org/10.19083/ ridu.2019.1078

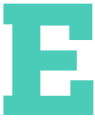
$\mathrm{n}$ los últimos años, se han venido ofreciendo programas educativos destinados a personas que cuentan con experiencia laboral, por parte de muchas universidades del ámbito privado (PQS. La voz de los emprendedores, 2016), utilizando para ello la modalidad semipresencial. Estas carreras podrían incluso ser culminadas en menor tiempo, si se les compara con la modalidad regular.

De acuerdo con el Instituto Nacional de Estadística e Informática (INEI), el $25 \%$ de los estudiantes universitarios tiene 23 años o más y cuenta con un empleo (INEI, 2010); de esta manera, un segmento importante de la población podría beneficiarse de la educación semipresencial. No obstante, dicha modalidad en el ámbito universitario presenta algunos desafíos como, por ejemplo, la poca motivación de los alumnos al momento de realizar las tareas virtuales (Sorbie, 2015), o el alto nivel de distracción ante la realización de dichas actividades (La Roche \& Flanigan, 2013). En la institución en la que se realizó este trabajo, se identificó una participación del $46 \%$ en las tareas virtuales por parte de los alumnos del programa semipresencial, ciclo 2018-enero (Dirección de Tecnologías para el Aprendizaje, 2018); esto indica que los estudiantes dejan de realizar una cantidad importante de actividades, las cuales contribuirían a conseguir los resultados de aprendizaje esperados; consecuentemente, participan de las clases presenciales sin revisar previamente el contenido online. Es por ello que se hace necesaria la identificación y aplicación de estrategias instruccionales que acrecienten la motivación de los alumnos para completar las tareas virtuales; este es el caso de las insignias digitales, las cuales son parte de las estrategias de gamificación, en las que se usan las características del juego para promover el aprendizaje (Kim, Song, Lockee, \& Burton, 2018); por ejemplo, a través de recompensas, retroalimentación e indicadores de progreso (Observatorio de Innovación Educativa, 2016).

Recientemente, el uso de las insignias digitales se ha incrementado (Universidad de Deusto, 2017). Por ejemplo, Reid, Paster y Abramovich (2015) realizaron un estudio en una universidad 
pública norteamericana con alumnos del primer año para analizar sus actitudes respecto del uso de las insignias digitales, el cual concluyó que estas tienen efectos diferentes de acuerdo con las características del estudiante; encontrándose que solo los alumnos con expectativas altas hacia el aprendizaje, incrementaron sus niveles de motivación intrínseca para obtener las insignias. Adicionalmente, Iosup y Epema (2014) realizaron una investigación en una universidad de los Países Bajos con 450 estudiantes de educación superior técnica, en la que se pretendió determinar los efectos de las insignias e indicadores de estado, entre otras estrategias de gamificación, sobre la tasa de aprobación, nivel de satisfacción, participación en clase y realización de tareas. Los resultados mostraron que más del $75 \%$ de alumnos aprobaron en su primer intento. Adicionalmente, se halló una correlación positiva entre la gamificación y la cantidad de estudiantes que aprobaron, así como también, con el completamiento de actividades voluntarias y de tareas.

Por último, Fajiculay, Parikh, Wright y Heck Sheehan (2017) realizaron una investigación en estudiantes de una universidad norteamericana, en la que se describió la percepción y motivación de los estudiantes de la carrera de farmacia en relación con las insignias digitales. Se encontró que el $53 \%$ de los estudiantes percibieron que éstas podrían serles de ayuda comprender a cabalidad el contenido de la asignatura. Asimismo, el 73\% de alumnos que consiguieron por lo menos una insignia, indicaron que éstas les ayudaron a mejorar sus niveles de confianza en el material de la asignatura; el 55\% afirmó que pudieron concentrarse en los resultados específicos del aprendizaje; el 64\% indicó que, a partir del uso de las insignias, tuvieron una comprensión más amplia de las competencias de la asignatura; por último, el $82 \%$ aseguró que las insignias digitales les sirvieron como complemento a las estrategias tradicionales de instrucción.

Otros estudios como los de Córdova Berona (2017), Abramovich, Schunn e Higashi (2013), Glover y Latif (2013) y Palazón-Herrera (2015) también dan cuenta de los efectos de las insignias digitales sobre la motivación y el rendimiento académico de estudiantes de educación secundaria y superior.

\section{Insignias digitales.}

Las insignias digitales son utilizadas para representar el dominio de una habilidad, así como el cumplimiento o desempeño en alguna una actividad (Shields \& Chugh, 2016); además, pueden ser estímulos motivadores que dirigen el comportamiento del alumno hacia un objetivo determinado. Usualmente se componen de una imagen en formato PNG, información en forma de metadatos y una página web, en la que se almacenan las, evidencias, criterios y los datos del emisor (Borrás, 2017). La parte más representativa de las insignias son los metadatos, que, además, las dotan de valor; estos hacen referencia a información sobre quién hizo entrega de la insignia, quién la ganó, en qué momento se obtuvo, cuáles fueron las condiciones para obtenerla, etc. (Masura, 2014). Además, como valor añadido, la representación visual de la insignia busca atraer al alumno. De esta manera, dicha imagen debería brindar información respecto del área o tema al que se hace referencia, de la institución, nivel de formación, o bien, de la descripción de la insignia (Borrás, 2018).

Hickey (2012) menciona que las insignias pueden cumplir algunas funciones como: (a) reconocimiento del aprendizaje; así pues, la posibilidad de acreditar aprendizajes informales es una de sus grandes ventajas (Hickey, 2012; The Mozilla Foundation and Peer 2 Peer University, 2012); (b) valoración del aprendizaje: las insignias contribuyen a la evaluación sumativa, la cual permite verificar que el aprendizaje ocurrió; a la evaluación formativa, la cual nos da luces sobre los aspectos a mejorar y a la evaluación de transformación, la cual busca cambios a la identidad del aprendiz (Baker, 2007; Davidson, 2011). (c) Motivación para el aprendizaje: estas pueden utilizarse para acrecentar el interés de los alumnos hacia un determinado tema, así como para orientarlo a completar actividades académicas, de forma que el aprendiz pueda reconocer qué procesos debe seguir para alcanzar un objetivo de aprendizaje (Gibson, Ostashewski, Flintoff, Grant, \& Knight, 2015).

No obstante, es importante reconocer que actualmente no existe claridad respecto del impacto real que tienen las insignias digitales en la motivación del aprendiz (Zellner, 2015). Para explicar el funcionamiento de las insignias digitales, podemos recurrir a la teoría de las metas motivaciona- 
les, la cual propone tres orientaciones que se pueden encontrar en cualquier situación educativa: la orientación al resultado, a la evitación y hacia el aprendizaje (Dweck \& Elliot, 2005; Huertas \& Montero, 2003). La investigación actual demuestra que, dentro de una misma orientación, pueden ponerse en juego diferentes metas de manera simultánea (Huertas, 2009). Se asume que, para que el estudiante busque conseguir una insignia determinada, la recompensa asociada a la insignia debe alinearse con la orientación motivacional del alumno; de lo contrario, hay una baja probabilidad de que el estudiante realice esfuerzos para conseguir alguna insignia.

El funcionamiento de las insignias digitales también puede ser explicado desde el aprendizaje de comportamientos; en este caso, desde el condicionamiento operante (Robson, Plangger, Kietzmann, McCarthy, \& Pitt, 2015); éste consiste en la presentación contingente de un estímulo a una determinada respuesta, generándose un incremento en la ocurrencia de este comportamiento (Leslie, 2005). Precisamente, una de las técnicas derivadas de este principio del aprendizaje es la economía de fichas. En esta técnica, los tokens o fichas son solo un conjunto de estímulos neutros que, al presentarse repetidamente y de manera contingente al estímulo reforzante, se transforman en entidades reforzantes en sí mismas (Doll, McLaughlin, \& Barretto, 2013).

Con lo descrito previamente, el presente estudio busca identificar los efectos de un programa de insignias digitales sobre la participación en las actividades virtuales y el desempeño académico de los alumnos del primer ciclo de una universidad privada, bajo la modalidad semipresencial. Como hipótesis de investigación, se propone que el programa de insignias digitales tendrá un efec- to significativo positivo sobre la actividad virtual de los alumnos del primer ciclo en modalidad semipresencial de una universidad privada, comparándoseles con un grupo de control.

\section{Método}

\section{Diseño.}

El alcance del presente estudio es explicativo, mientras que el diseño empleado fue cuasi experimental, con pos prueba y grupo control (Hernández, Fernández, \& Baptista, 2014). Así pues, al concluirse la manipulación de la variable independiente, se midieron los niveles de las variables dependientes en ambos grupos.

\section{Participantes.}

Los participantes fueron los alumnos del primer ciclo de una universidad privada con sede en Lima, todos ellos cursaban la asignatura Inglés I bajo la modalidad semipresencial. Respecto del grupo experimental, participaron 14 hombres y cuatro mujeres, haciendo también un total de 18 estudiantes. Considerando al grupo control, participaron 13 hombres y cinco mujeres, haciendo un total de 18 estudiantes. La asignación de estudiantes en ambos grupos se realizó a través del emparejamiento, el cual es un método para hacer equivalentes a ambos grupos a partir de una determinada variable (Hernández et al., 2014). En este caso, la variable utilizada para emparejar a los estudiantes fue el nivel de inglés, escogiéndose la misma cantidad de estudiantes, tanto para el grupo control, como para el experimental, respecto del cuartil en el que se ubican dicha variable (ver Tabla 1).

Tabla 1

Estudiantes clasificados de acuerdo con su grupo y con el cuartil obtenido en el nivel de inglés

\begin{tabular}{|l|c|c|c|c|c|}
\hline \multirow{2}{*}{} & \multicolumn{2}{|c|}{ Sexo } & \multicolumn{3}{c|}{ Cuartil } \\
\hline Grupo & Hombres & Mujeres & 1 & 2 & 3 \\
\hline Control & 13 & 5 & 6 & 8 & 12 \\
\hline Experimental & 14 & 4 & 6 & 8 & 12 \\
\hline Total & 27 & 9 & 12 & 16 & 24 \\
\hline
\end{tabular}




\section{Técnicas para la recolección de datos.}

Las variables independientes son el rendimiento académico y la participación en las actividades virtuales. En el primer caso, la medición se realizó a través del promedio de notas obtenidas por los estudiantes, el cual consistió en dos prácticas y un trabajo final. En el caso de la participación en las actividades virtuales, se midió a través del conteo de tareas virtuales completadas en cada grupo.

La recolección de las variables dependientes se realizó a través de la observación; específicamente, utilizando la medición de productos permanentes. Es decir, se recogió información sobre los resultados o rastros de evidencia del comportamiento emitido (McKay, 2008). Una de las ventajas más resaltantes de esta técnica es la facilidad con la que se puede recolectar la información, así como la objetividad de los productos tangibles del comportamiento.

\section{Procedimiento.}

Primero se diseñó el programa de insignias y, luego, se llevó a cabo su implementación en el sistema de gestión del aprendizaje (LMS) Canvas, a través de la aplicación Badgr. Además, la muestra de estudiantes fue conseguida con el apoyo de la coordinación del curso de Inglés I. Se escogió este curso debido a la elevada cantidad de matriculados en relación con las demás asignaturas. A continuación, se emparejó aleatoriamente a los estudiantes para asignarlos tanto al grupo control y experimental, con base en el cuartil en el que se encontraban en su nivel de inglés; para medir ello, se utilizó una prueba análoga a las evaluaciones aplicadas a los estudiantes durante el transcurso de la asignatura, obteniéndose así 18 participantes para el grupo experimental y la misma cantidad para el grupo control.

El curso se dictó en el segundo periodo del 2018 y duró nueve semanas para ambos grupos. Es importante mencionar que el mismo profesor tuvo a su cargo al grupo control y experimental, los cuales empezaron sus clases en el mismo periodo. Al inicio del curso, se aplicó una encuesta a los estudiantes de ambos grupos, con la finalidad de obtener datos sociodemográficos. Durante el desarrollo de las clases, los datos relacionados con la actividad de los estudiantes en el LMS fueron recabados por el área de evaluación y monitoreo de la institución.

Para ambos grupos, el profesor se ciñó a un conjunto de lineamientos relacionados con el uso del
LMS, que incluyeron el envío de anuncios, participación en videoconferencias y en un foro destinado a absolver dudas.

El programa de insignias consistió en el completamiento de un conjunto de actividades virtuales, con lo cual el estudiante se hacía acreedor de una insignia, la cual se podía visibilizar en el LMS. Cada una de las insignias implicaba el completamiento de un máximo de tres actividades virtuales, las cuales incluyeron el envío de comentarios en dos foros, seis entregas de ejercicios y seis autoevaluaciones, como se observa en la tabla 2.

Al conseguir tres o cuatro insignias, cada estudiante se hizo acreedor de otra insignia de nivel superior, que podía intercambiar por un punto de bonificación sobre la nota obtenida en alguna de las prácticas calificadas. Al finalizar la asignatura, el profesor asignó dicha puntuación en el registro de notas.

De manera complementaria, el docente envío anuncios a través del LMS, incluyendo un mensaje de congratulación a los estudiantes que alcanzaron alguna de las insignias y un recordatorio de aquellas insignias por recolectar. Es importante mencionar que, durante las clases presenciales, se reforzó el uso de las insignias a través de felicitaciones y mensajes de motivación.

\section{Resultados}

Una vez concluida el acopio de los datos, se utilizó el software IBM SPSS en su versión 25. Con los datos obtenidos, se procedió a corroborar el cumplimiento de los requisitos necesarios para realizar la comparación de muestras independientes con una prueba paramétrica (Coolican, 2014). Primero se verificó el supuesto de normalidad en la puntuación en la participación en las actividades virtuales a través del estadístico Shapiro-Wilks, el cual se usó debido a que la muestra incluyó a menos de cincuenta individuos, desconociéndose la media y varianza de la población (Díaz, 2009). Los resultados indican que los puntajes de participación del grupo experimental se aproximan a una distribución normal; mientras que, en el caso del grupo control, los puntajes no se aproximan a dicha distribución, como se observa en la Tabla 3. 
Tabla 2

Distribución de las insignias digitales

\begin{tabular}{|c|c|c|}
\hline $\begin{array}{l}\text { Semana de } \\
\text { clases }\end{array}$ & Actividad realizada por el alumno & $\begin{array}{l}\text { Denominación } \\
\text { de la insignia }\end{array}$ \\
\hline \multirow{2}{*}{1} & Envío de un comentario en el foro de presentación “Talking about me”. & Me and you \\
\hline & Completamiento de la primera autoevaluación. & Be nice \\
\hline \multirow{3}{*}{2} & $\begin{array}{l}\text { Envío del trabajo } 2 \text { - ficha de aplicación del tema "Demonstrative Pronouns } \\
\text { and Plural nouns". }\end{array}$ & \multirow[t]{2}{*}{ People and things } \\
\hline & Completamiento de la segunda autoevaluación. & \\
\hline & $\begin{array}{l}\text { Obtención de las siguientes insignias: } \\
\text { - Me and you } \\
\text { - Be nice } \\
\text { - People and things }\end{array}$ & In english, please I \\
\hline 3 & $\begin{array}{l}\text { Envío del trabajo individual } 3 \text { - ficha de aplicación del tema } \\
\text { "Possessive adjectives". }\end{array}$ & My house is your house \\
\hline \multirow{2}{*}{4} & Envío de un comentario en el foro "My familiy" & \multirow{2}{*}{ Happy family } \\
\hline & Completamiento de la tercera autoevaluación. & \\
\hline \multirow[b]{3}{*}{5} & Envío del trabajo 5 - ficha de aplicación del tema "There is -There are". & \multirow{2}{*}{ Out and about } \\
\hline & Completamiento de la cuarta autoevaluación. & \\
\hline & $\begin{array}{l}\text { Obtención de las siguientes insignias: } \\
\text { - My house is your house } \\
\text { - Happy family } \\
\text { - Out and about }\end{array}$ & In english, please II \\
\hline \multirow{2}{*}{6} & Envío de la presentación del primer avance del trabajo final. & \multirow{2}{*}{ Love life } \\
\hline & Completamiento de la quinta autoevaluación. & \\
\hline 7 & $\begin{array}{l}\text { Envío del trabajo individual } 7 \text { - ficha de aplicación del tema } \\
\text { "Adverbs of frequency". }\end{array}$ & Work and play \\
\hline \multirow[b]{3}{*}{8} & Envío de la presentación del segundo avance del trabajo final. & Yes, I can \\
\hline & Completamiento de la sexta autoevaluación. & Close to the top \\
\hline & $\begin{array}{l}\text { Obtención de las siguientes insignias: } \\
\text { - Love life } \\
\text { - Work and play } \\
\text { - Yes, I can } \\
\text { - Close to the top }\end{array}$ & In english, please III \\
\hline
\end{tabular}

Tabla 3

Estadísticos respecto de la participación en las actividades virtuales

\begin{tabular}{|l|c|c|c|c|c|c|c|c|}
\hline Grupo & $\boldsymbol{M}$ & $\boldsymbol{M e}$ & $\boldsymbol{D E}$ & $\boldsymbol{M i n}$ & $\boldsymbol{M a x}$ & $\boldsymbol{S W}$ & $\boldsymbol{U}$ & $\boldsymbol{P}_{\boldsymbol{s}}$ \\
\cline { 1 - 7 } Experimental & 9.22 & 9 & 2.34 & 5 & 14 & $p>.05$ & \multirow{2}{*}{$16.00^{* *}$} & 4.57 \\
\hline Control & 5.11 & 5 & 0.90 & 2 & 6 & $p<.05$ & \\
\hline
\end{tabular}

Nota: $M=$ media; $M e=$ mediana; $D E=$ desviación típica o estándar; $M i n=v a l o r$ mínimo; $M a x=$ valor máximo; $S W=S h a p i r o-W i-$ lks. U: U de Mann-Whitney. $P_{s}$ : Probabilidad de superioridad. 
Tabla 4

Estadísticos descriptivos para la cantidad de insignias conseguidas por el grupo experimental

\begin{tabular}{|l|c|c|c|}
\hline Insignias & $\boldsymbol{f}$ & Proporción alcanzada & Proporción restante \\
\hline Posibles & 252 & 45.24 & 55.76 \\
\hline Obtenidas & 114 & \\
\hline
\end{tabular}

Tabla 5

Estadísticos respecto del rendimiento académico

\begin{tabular}{|c|c|c|c|c|c|c|c|c|}
\hline Grupo & $M$ & $M e$ & $D E$ & Min & $\operatorname{Max}$ & sw & $\boldsymbol{U}$ & $\boldsymbol{P}_{s}$ \\
\hline Experimental & 17.39 & 17 & 1.19 & 15 & 19 & $p<.05$ & \multirow{2}{*}{100.5} & \multirow{2}{*}{0.60} \\
\hline Control & 14.61 & 17 & 4.63 & 1 & 20 & $p<.05$ & & \\
\hline
\end{tabular}

Nota: $M=$ media Me= mediana $D E=$ desviación típica o estándar; $M i n=$ valor mínimo; $M a x=$ valor máximo; $S W=$ Shapiro-Wilks. U: U de Mann-Whitney. Ps: Probabilidad de superioridad.

Teniendo en cuenta ello, se decidió utilizar una prueba no paramétrica; en este caso, la U de Mann-Whitney. A partir de estos resultados, se rechaza la hipótesis nula, la cual indica que las medias de ambas secciones son iguales, pues la probabilidad es <.001, menor al .05.

Adicionalmente, se identificó el tamaño del efecto, el cual viene a ser la dimensión de la relación o diferencia encontrada en la investigación (Howitt \& Cramer, 2017); también, se ha definido como la medida de la fuerza de la diferencia de los promedios u otros valores (Creswell, 2013a; Alhija \& Levy, 2009; Cortina, 2003, como se citó en Hernández, et al., 2014). Ya que en esta investigación se trabajó con un grupo control, se hizo uso de la fórmula de Glass (Castillo, 2014), la cual nos permite afirmar que el tamaño del efecto tiene una dimensión muy grande, debido a que $d=4.57$.

Por otro lado, se identificó también la cantidad de insignias conseguidas, la cual fue de 114 . Al comparar este número con la cantidad total de insignias esperadas, se obtiene un porcentaje de 45.24 participaciones. Además, se realizó la comparación entre el rendimiento académico en ambos grupos; considerándose también el uso de la prueba U de Mann-Whitney (Coolican, 2014), debido a que la distribución de las muestras no fue normal, como se visualiza en la tabla 5.

Como puede apreciarse, la media de calificacio- nes del grupo experimental no difiere significativamente de la media de calificaciones del grupo control, con una significancia del 5\%. No obstante, se encontró un tamaño del efecto moderado, debido a que $d=0.60$. Estos resultados en apariencia discrepantes pueden explicarse debido al tamaño de la muestra, lo cual disminuye la probabilidad de encontrar diferencias estadísticamente significativas.

\section{Discusión}

A partir de los resultados obtenidos se pudo verificar un efecto estadísticamente significativo en la participación en las actividades virtuales realizadas por los alumnos del grupo experimental, comparándolos con los alumnos del grupo de control; asimismo, el tamaño de efecto es grande.

Estos hallazgos concuerdan de manera parcial con el estudio de Iosup y Epema (2014) en el que se encontró una correlación entre el programa de gamificación con un aumento en el porcentaje de estudiantes aprobados y su completamiento de actividades complementarias. Además, se encuentra en la misma línea de otros estudios como el de Abramovich, Schunn e Higashi (2013), quienes indican que las insignias tienen un efecto positivo sobre los niveles de motivación en los es- 
tudiantes, lo cual podría traducirse en un mayor cumplimiento de las actividades. No obstante, en el presente trabajo no se ha evaluado específicamente la motivación, por lo que sería necesario corroborar este punto con otras investigaciones.

Este impacto encontrado sobre la participación podría explicarse por los principios de aprendizaje conductual, particularmente, el condicionamiento respondiente $\mathrm{y}$ operante (Robson et al., 2015). Si bien las insignias digitales tienen inicialmente poco o ningún valor, adquieren las propiedades de los reforzadores luego de ser asociadas de manera contingente. De esta manera, el conseguir una insignia digital es reforzante para el alumno, aumentando la probabilidad de realizar los comportamientos necesarios para volver a conseguirlas.

Por otro lado, no se hallaron diferencias estadísticamente significativas en el desempeño académico de los alumnos del grupo experimental y del grupo control. No obstante, al analizar el tamaño del efecto, se puede afirmar que la magnitud de la diferencia de medias es moderada, por lo que se infiere que el programa tuvo cierto impacto sobre el rendimiento de los estudiantes, aunque existe una alta probabilidad de que esta diferencia se deba al azar. Con los resultados obtenidos no se puede establecer con certeza una relación entre la participación en las actividades calificadas y la calificación obtenida por los estudiantes, por lo que se requeriría de estudios adicionales que abarquen tamaños de muestra superiores, para corroborar si, con más actividades virtuales, los alumnos tienen una mayor práctica de los contenidos de la asignatura, lo cual se reflejaría en sus notas.

Respecto a las insignias digitales, se consiguieron en promedio 114 de las 252 posibles, lo que equivale al $45.24 \%$; en consecuencia, hubo un $55.21 \%$ de insignias que no fueron conseguidas. Esto puede haber ocurrido debido a que no todas las tareas virtuales tienen un impacto en el promedio final, a excepción de la bonificación brindada a partir de las insignias digitales. De esta manera, un estudiante que no tuviera la necesidad de conseguir dicha bonificación, no se encontraría motivado para completar estas tareas virtuales.
Esto se explica a partir de la teoría de las metas motivacionales (Huertas, 2009); así pues, la priorización de las actividades virtuales varía de acuerdo con la meta motivacional del alumno: si un estudiante se orienta al resultado y busca exclusivamente aprobar, solo necesitaría llevar a cabo las acciones mínimas necesarias para ello y, si ya tuviera la puntuación necesaria, no necesitaría conseguir ninguna insignia digital adicional. Se infiere que, de acuerdo con la meta que un alumno tenga respecto de la asignatura, llevará a cabo distintas conductas destinadas a alcanzar dicha meta, lo cual concuerda con lo identificado por Reid et al. (2015), quienes indican que las insignias tienen efectos diferentes de acuerdo con las características del estudiante. En su estudio se halló una percepción favorable de las insignias en el curso de inglés en la mayoría de alumnos; no obstante, los niveles motivacionales intrínsecos para obtener las insignias se acrecentaron solo para los aprendices con una expectativa elevada hacia el aprendizaje. Además, estos hallazgos se encuentran en una dirección similar a lo encontrado por Glover y Latif (2013), quienes indicaron que, si muchos participantes pueden adquirir una determinada insignia, el efecto de esta puede diluirse.

Estos resultados permiten corroborar la efectividad de las insignias digitales como elementos motivadores en la participación de los alumnos en actividades académicas virtuales bajo la modalidad semipresencial, específicamente, en el aprendizaje del inglés. Sin embargo, la investigación tuvo algunas limitaciones; por ejemplo, no se realizó la evaluación de otras variables relevantes que están relacionadas con la participación académica, como es el caso de la motivación. Otra limitación tiene que ver con el tamaño de la muestra, por lo que los resultados obtenidos respecto del rendimiento académico no son generalizables.

Se recomienda realizar investigaciones en cursos de diferentes áreas para comprobar la generalización de estos resultados a la instrucción de otro tipo de contenidos. Además, es importante ampliar la muestra de investigación para poder incrementar la probabilidad de obtener resultados más consistentes. 


\section{Referencias}

Abramovich, S., Schunn, C. D., \& Higashi, R. M. (2013). Are badges useful in education?: it depends upon the type of badge and expertise of learner. Education Tech Research Dev, 61(2), 217-232. http://doi.org/10.1007/ s11423-013-9289-2

Baker, E. L. (2007). 2007 presidential address-The end (s) of testing. Educational Researcher, 36(6), 309-317. https://doi.org/10.3102/0013189X07307970

Borrás, O. (2017). Insignias digitales como acreditación de competencias en la Universidad. Universidad Politécnica de Madrid, Gabinete de Tele-Educación, Madrid.

Borrás, O. (2018). Buenas prácticas UPM con insignias digitales. Universidad Politécnica de Madrid, Gabinete de tele-educación, Madrid. Recuperado de http://oa.upm.es/51833/1/Buenas\%20practicas\%20 UPM\%20con\%20insignias\%20digitales.pdf

Castillo Blanco, R. (2014). Reporte del tamaño del efecto en los artículos de tres revistas de psicología peruanas en los años 2008 al 2012. (Tesis de Licenciatura). Universidad Nacional Mayor de San Marcos, Lima.

Coolican, H. (2014). Research Methods and Statistics in Psychology (Sixth ed.). New York: Rouledge. https:// doi.org/10.4324/9780203769669

Córdova Berona, H. A. (2017). Entrega de medallas por el esfuerzo e insignias por alcanzar el logro. Conferencia en el $4^{\circ}$ Congreso de Innovación Educativa. Universidad Tecnológica del Perú.

Davidson, C. N. (2011, Noviembre 14). Could badges for lifelong learning be our tipping point? Recuperado de https://www.hastac.org/blogs/cathy-davidson/2011/11/14/could-badges-lifelong-learning-be-our-tipping-point

Díaz, A. (2009). Diseño estadístico de experimentos (Segunda ed.). Antioquia: Universidad de Antioquia.

Dirección de Tecnologías para el Aprendizaje. (2018). Reporte de seguimiento semanal - CGT UTP 2018 enero. Lima, Perú.

Doll, C., McLaughlin, T. F., \& Barretto, A. (2013). The Token Economy: A Recent Review and Evaluation. International Journal of Basic and Applied Science, 2(1), 131-149.

Dweck, C., \& Elliot, D. S. (2005). Handbook of competence and motivation. New York: Guilford Press.

Fajiculay, J. R., Parikh, B. T., Wright, C. V., \& Heck Sheehan, A. (2017). Student perceptions of digital badges in a drug information and literature evaluation course. Currents in Pharmacy Teaching and Learning. http://doi.org/10.1016/j.cptl.2017.05.013

Gibson, D., Ostashewski, N., Flintoff, K., Grant, S., \& Knight, E. (2015). Digital badges in education. Educ Inf Technol, 20(2), 403-410. https://doi.org/10.1007/ s10639-013-9291-7

Glover, I., \& Latif, F. (2013). Investigating Perceptions and Potential of Open Badges in Formal Higher Education. In J. Herrington, A. Couros, \& V. Irvine (Eds.), Proceedings of World Conference on Educational Multimedia, Hypermedia and Telecommunications (pp. 1398-1402). Chesapeake.

Hernández, R., Fernández, C., \& Baptista, P. (2014). Metodología de la investigación (Sexta ed.). México D.F.: Mcgraw-hill / Interamericana editores, s.a. de c.v.

Hickey, D. (2012, Junio 10). Digital badges as transformative assessment. Retrieved from http://remediatingassessment.blogspot.com/2012/06/digital-badges-as-transformative.html

Howitt, D., \& Cramer, D. (2017). Research Methods in Psychology (Fifth ed.). Harlow: Pearson Education Limited.

Huertas, J. A. (2009). Aprender a fijarse metas: Nuevos estilos motivacionales. In J. I. Pozo, \& M. D. Pérez Echeverría (Eds.). Madrid: Ediciones Morata.

Huertas, J. A., \& Montero, I. (2003). Motivación en el aula. In E. Fernández Abascal, M. P. Jiménez, \& M. D. Martín, Emoción y Motivación: la adaptación humana. Madrid: UNED-Fundación.

INEI. Instituto Nacional de Estadística e Informática. (2010). II Censo Nacional Universitario 2010. Sistema de Consulta de Datos. Recuperado de https://webinei. inei.gob.pe/anda_inei/index.php/catalog/264

Iosup, A., \& Epema, D. (2014). An Experience Report on Using Gamification in Technical Higher Education. SIGCSE '14 Proceedings of the 45th ACM technical symposium on Computer science education, 27-32. https://doi.org/10.1145/2538862.2538899

Kim, S., Song, K., Lockee, B., \& Burton, J. (2018). Gamification in Learning and Education. Enjoy Learning Like Gaming. Cham: Springer. https://doi.org/10.1007/9783-319-47283-6

La Roche, C. R., \& Flanigan, M. A. (2013). Student Use of Technology in Class: Engaged or Unplugged? Journal of College Teaching \& Learning, 10(1), 47-54. https:// doi.org/10.19030/tlc.v10i1.7537

Leslie, J. C. (2005). Principles of Behavioral Analysis. New 
York: Psychology Press.

Masura, S. (2014). Digital Badges. Cherry Lake.

McKay, D. (2008). Handbook of Research Methods in Abnormal and Clinical Psychology. California: SAGE.

Observatorio de Innovación Educativa. (2016). EduTrends. Gamificación. Reporte, Tecnológico de Monterrey.

Palazón-Herrera, J. (2015). Motivación del alumnado de educación secundaria a través del uso de insignias digitales. Opción(1), 1059 - 1079.

PQS. La voz de los emprendedores. (2016, Noviembre 12). Carreras universitarias para gente que trabaja. $P Q S$. La voz de los emprendedores. Recuperado de https://www.pqs.pe/actualidad/noticias/carreras-universitarias-para-gente-que-trabaja

Reid, A. J., Paster, D., \& Abramovich, S. (2015). Digital badges in undergraduate composition courses: effects on intrinsic motivation. J. Comput, 2(4), 377-398. https://doi.org/10.1007/s40692-015-0042-1

Robson, K., Plangger, K., Kietzmann, J. H., McCarthy, I., \& Pitt, L. (2015). Is it all a game? Understanding the principles of gamification. Business Horizon, 58, 411-420. https://doi.org/10.1016/j.bushor.2015.03.006

Salmerón, H., Gutiérrez, C., Salmerón, P., \& Rodríguez, S. (2011). Metas de logro, estrategias de regulación y rendimiento académico en diferentes estudios universitarios. Revista de Investigación Educativa,
29(2), 467-486.

Shields, R., \& Chugh, R. (2016). Digital badges - rewards for learning. Educ Inf Technol. https://doi.org/10.1007/ s10639-016-9521-x

Sorbie, J. I. (2015). Exploring Teacher Perceptions of Blended Learning. Doctoral Study , Walden University. Recuperado de https://scholarworks.waldenu.edu/ cgi/viewcontent.cgi?article=2969\& context=disser tations

The Mozilla Foundation and Peer 2 Peer University. (2012) Open Badges for Lifelong Learning. Exploring an open badge ecosystem to support skill develop ment. Recuperado de https://wiki.mozilla.org/images/b/b1/OpenBadges-Working-Paper_092011.pdf

Universidad de Deusto. (2017, Noviembre 07). Motiva a tus estudiantes mediante el uso de insignias digitales en ALUD. Recuperado de https://blogs.deusto.es/ aprender-ensenar/motiva-a-tus-estudiantes-mediante-el-uso-de-insignias-digitales-en-alud/

Zellner, A. (2015). 21st Century Rewards: A Case Study of Khan Academy and Digital Badges from an Educational Psychology Perspective. Society for In formation Technology \& Teacher Education International Conference (pp. 1899-1906). Las Vegas: Association for the Advancement of Computing in Education (AACE).

RIDU / Revista Digital de Investigación en Docencia Universitaria / e-ISSN: 2223-2516

(C) Los autores. Este artículo es publicado por la Revista Digital de Investigación en Docencia Universitaria del Área de Institutional Research and Effectiveness de la Dirección de Aseguramiento de la Calidad, Universidad Peruana de Ciencias Aplicadas. Este es un artículo de acceso abierto, distribuido bajo los términos de la LicenciaCreativeCommons Atribución-CompartirIgual 4.0 Internacional.(http://creativecommons.org/licenses/by-sa/4.0/), que permite el uso no comercial, distribución y reproducción en cualquier medio, siempre que la obra original sea debidamente citada. 


\section{Apéndices}

Tabla 6

Insignias digitales

\begin{tabular}{|c|c|}
\hline Nombre de la insignia & Representación gráfica \\
\hline Me and you & \\
\hline Be nice & \\
\hline People and things & \\
\hline In english, please I & \\
\hline My house is your house & \\
\hline Happy family & \\
\hline
\end{tabular}






